\title{
Design of a Compact Tunable Wavelength Division Multiplexing Photonic Phased Array Switch Using Nano Electromechanical Systems on a Silicon-on- Insulator Integration Platform
}

\author{
Ali A. Hussein, Sawsan A. Majid and Trevor J. Hall \\ Center for Research in Photonics at the University of Ottawa, Ottawa, Canada K1N6N5, ahuss052@uottawa.ca
}

\begin{abstract}
We present the design of a novel $4 \times 4$ photonic phased array switch element with the capability to actively tune WDM pass-bands. The switch element uses four NEMS-operated phase shifters located on the two longitudinal sides of the structure; offering advantages of compact footprint and lowpower consumption. All components of the switch element are designed to state of the art parameters. The design is verified using the finite difference mode three-dimensional full-vectorial solver of the simulation tool FIMMPROP and utilizing custom numerical analysis.
\end{abstract}

\section{INTRODUCTION}

The number of reports in the literature of the design of advanced silicon on insulator (SOI) photonic integrated circuits (PICs) has been increasing reflecting the growing appreciation by designers of the tight light confinement of the high refractive index between the silicon guiding ridge and the surrounding cladding that offers compact size and low loss. The NEMS-operated phase shifters of cascaded under-etched slot waveguides introduced by Acoleyen et al [1] has paved the way to produce ultra-small size low-power consumption voltage controlled active SOI devices. Paiam and MacDonald [2] presented designs of wavelength division multiplexed (WDM) wavelength routing (de)multiplexers using splitter/combiner multimode interference (MMI) couplers with path length imbalanced interconnection networks between them. We presented the concept of phased array switches in [3] where a novel $2 \times 2$ Integrated Photonic Phased Array Switch (IPPAS) element with path length differences is used to associate the shift in the resulted output wavelength interferogram patterns with the phase shift differences achieved by the NEMS-operated under-etched slot waveguides. In this work, we present the use of the NEMSoperated phase shifters and path length differences in a $4 \times 4$ IPPAS element achieving tunable WDM wavelength routing functionality.

IPPAS elements are compact phased array Mach-Zehnder (MZI) interferometer type switches with any number of multi inputs and outputs. They may be implemented by concatenating two input/output (splitter/combiner) MMI couplers using an interconnection network composed of NEMS-operated phase shifters, path length difference, nanowires waveguides, and adiabatic tapers. $2 \times 2 \mathrm{MMI}$ couplers may also be used to implement waveguide crossovers to bring the inner paths to the outer longitudinal sides of the phased array to ease the layout of the phase shifters.

The schematic diagram of the $4 \times 4$ tunable WDM-IPPAS element shown in Fig. 1 depicts the novel features of this family of PICs. The pitch size between the port centers of all of the MMI couplers is the same enabling the nanodimensions NEMS-operated phase shifters to be aligned with the ports of the interconnected MMI couplers and eliminating the need to use waveguide loops to compensate for path length imbalance. One of the two silicon beams of each phase shifter is electrically isolated and is biased from one (exterior) side, whereas the other is excited through the rest of the system silicon structure taken as the electrical ground. This avoids the need to use waveguide crossovers and facilitates the electrical probing to the chip compared with analogous conventional switches. Edge couplers, as shown in Fig. 1, or grating couplers can be used to couple the light in/out of the PIC. The $2 \times 2$ MMI coupler crossovers at the output ports facilitate coupling the ground to the structure. These can be eliminated in case of the use of grating couplers. The overall structure is symmetrical and losses are well-balanced for all paths.

\section{NEMS-OPERATED PHASE SHIFTERS}

The NEMS-operated phase shifter shown schematically in Fig. 2 follows the design procedure disclosed in [3]. It consists of 15 cascaded under-etched slot waveguides capable of achieving up to $180^{\circ}$ of phase shift difference; transitions slot couplers that are $96.7 \%$ efficient according to simulations and the probing structure to bias the electrically isolated silicon beam of the phase shifter. The overall excess loss of the phase shifter is $1.1 \mathrm{~dB}$ according to simulation and it has a total length of only $349 \mu \mathrm{m}$.

Each under-etched slot waveguide has a length of $5.4 \mu \mathrm{m}$; an operational voltage up to $15 \mathrm{~V}$, worst case pull-in voltage (based on hinged boundary conditions) close to $17.7 \mathrm{~V}$ and a resonant frequency of the free-standing silicon beams around $50 \mathrm{MHz}$. The design is conducted by simulating the effective index of the under-etched slotted nanowire waveguide versus the slot width and then using these data in the nanoelectromechanical nonlinear numerical model presented in [1] and [3]. 


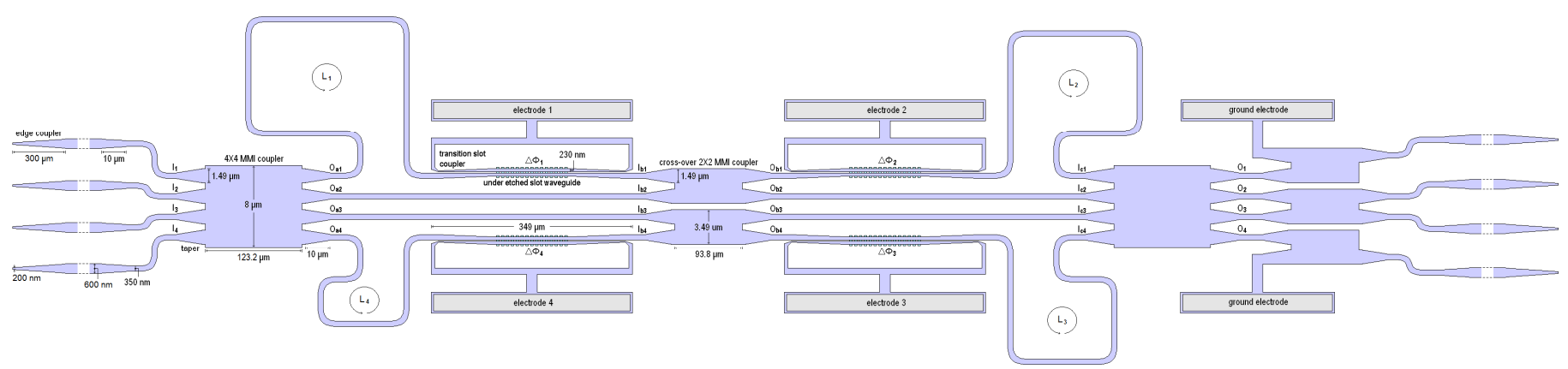

Fig. 1. Schematic diagram of the $4 \times 4$ tunable WDM-IPPAS element.



Fig. 2. Schematic diagram of the NEMS-operated phase shifter.

\section{MMI COUPLERS}

The designs of the $-3 \mathrm{~dB}$ and crossover $2 \times 2$ MMI couplers are justified in [3]. A maximum width of $2 \mu \mathrm{m}$ is reserved to each port of the $-3 \mathrm{~dB} 2 \times 2 \mathrm{MMI}$ coupler. An access port width of $1.49 \mu \mathrm{m}$ for which is shown to null the imbalance between the two outputs. We use the same width settings for the design of the $-6 \mathrm{~dB} 4 \times 4$ MMI coupler; setting the total coupler width to $8 \mu \mathrm{m}$. The simulation of the imbalances between outputs $1 / 4$ and $2 / 3$ due to both inputs 1 and 3 and the resulted associated excess losses versus the length of the coupler $L_{c}$ is depicted in Fig. 3. The optimized excess loss $L_{\text {opt }}$ is the variance of the excess losses $L_{1}$ and $L_{3}$ due to inputs 1 and 3 , respectively. At the coupler length $123.2 \mu \mathrm{m}$, a maximum imbalance of only $0.08 \mathrm{~dB}$ and minimum $L_{\text {opt }}=0.26 \mathrm{~dB}$ are found. The simulated optical power distribution inside the designed coupler is depicted in Fig. 4 for both inputs 1 and 3.

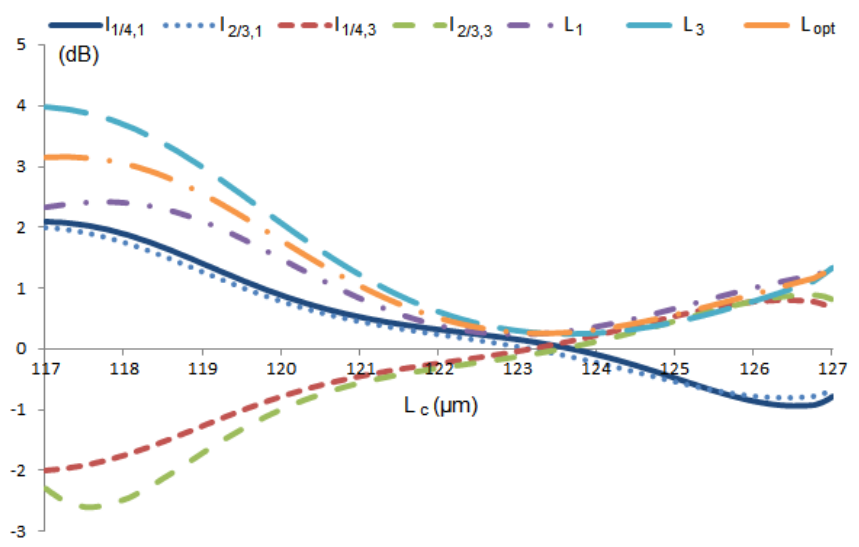

Fig. 3. Simulation of imbalances and excess losses for the $4 \times 4$ MMI coupler.

\section{WdM DESIGN OptIMIZATION}

The wavelength response of the magnitude and phase of the transmission between the ports of each MMI coupler and the wavelength response of the effective index of the path difference nanowires are simulated and used in custom software to optimize the path length differences: $L_{1}-L_{4}=150$ $\mu \mathrm{m}, L_{2}-L_{4}=100.12 \mu \mathrm{m}$ and $L_{3}-L_{4}=50 \mu \mathrm{m}$; resulting in the desired WDM response shown in Fig. 5. When applying progressive phase shift through the active phase shifters, the transmission interferogram patterns are steered in the wavelength domain by an average shift $0.0263 \mathrm{~nm} / \mathrm{deg}$.
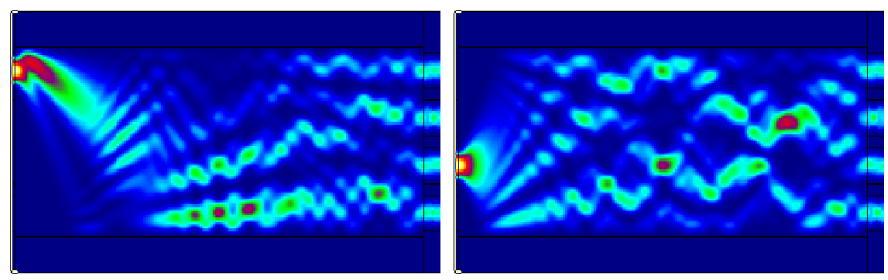

Fig. 4. Simulated optical power distribution inside the $4 \times 4$ coupler.

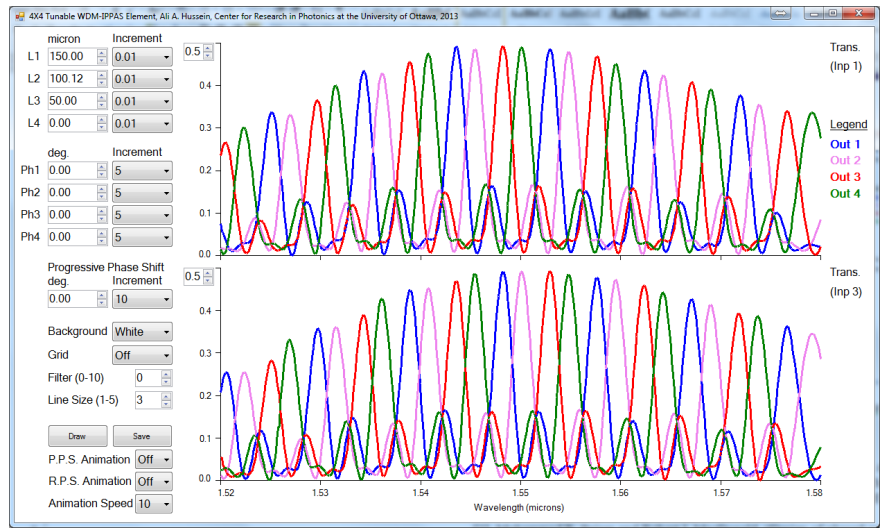

Fig. 5. Transmission interferogram response for inputs 1 (top) and 3 (bottom).

\section{SUMMARY}

The design of a novel $4 \times 4$ photonic phased array switch with tunable WDM pass-bands capability has been verified.

\section{REFERENCES}

[1] K. Van Acoleyen, J. Roels, P. Mechet, T. Claes, D. Van Thourhout, and R. Baets, "Ultracompact phase modulator based on a cascade of NEMSoperated slot waveguides fabricated in silicon-on-insulator," IEEE Photonics Journal, vol. 4, no. 3, , pp.779-788, June 2012.

[2] Mohammad R. Paiam and Robert I. MacDonald, "Design of phased-array wavelength division multiplexers using multimode interference couplers," Applied Optics, vol. 36, no. 21, pp. 5097- 5108, July 1997.

[3] Ali A. Hussein, Sawsan A. Majid, and Trevor J. Hall, "Design, simulation and fabrication of nano-scale silicon-on-insulator (SOI) integrated photonic phased array switch elements (IPPAS) utilizing nano electromechanical systems (NEMS)," 15'th Photonics North 2013 Conference, Ottawa, Canada, June 2013. 